\title{
ROBOTIC THEATRE: COMPARATIVE ANALYSIS OF HUMAN AND MECHANIZED ACTIVITIES IN THE CREATIVE PROCESS
}

\author{
Tetiana SOVHYRA \\ Variety Art Department, Faculty of Variety Art Directing, Kyiv National University of Culture and Arts, \\ Konovalets str. 36, 01601 Kyiv, Ukraine
}

Received 12 September 2020; accepted 23 December 2020

\begin{abstract}
The article systematizes and analyzes the existing experience of organizing the creative process in a robotic theater. The author explores the robotic theater phenomenon, the artificial intelligence technology possibilities to function in the stage space. The article provides a comparative analysis of human and mechanized interaction in the stage space. The methodological basis of the research is a combination of several methods: analytical - for accounting for historical and fictional literature; theoretical and conceptual method - for analyzing the conceptual and terminological system of research and identifying the specifics of introducing the artificial intelligence technology in creative process; comparative-typological - to compare the peculiarities of the functioning of mechanized "actors" with the acting skills of human performers. The article explores the threat perception and uncanny valley concepts to study the perception of a robot-actor by an audience. The author examines the process of human interaction with a robotic body: from the moment of interest, interaction to the moment of rejection of the robot by a person (audience).
\end{abstract}

Keywords: actor, artificial intelligence, creativity, digital technology, robot, theater.

\section{Introduction}

Since antiquity, the mankind has been constantly in search of unique technologies that would simplify production and creative activities. Let us recall Golem, the character of Jewish mythology, which, according to a legend, was created by Cabbalist rabbis and programmed to perform work too difficult for humans.

Back in 1495, Leonardo da Vinci makes sketches of a robot that is similar in structure to a human being and has to functionally imitate human activities. The scientist calls his mechanism Leonardo's robot, or the humanoid automaton. However, that sketch was lost for a long time, as well as many other essays of the inventions of the scientist and artist da Vinci.

Since then, the idea of creating robotic technology that facilitates human activity never ceased to attract scientists. The concept of aesthetic computing appears in the mirror of

\footnotetext{
*Corresponding author. E-mail: stisovgyra@gmail.com
} 
scientific criticism, which is a systematic approach to studying the relationship between innovative digital technologies and art, "applying the theory and practice of art to computing" (Fishwick, 2006, pp. 8-12). According to these studies, digital and computational technologies influence art and aesthetics, expanding our perceptual dimensions, while art and aesthetics also influence digital technologies, expanding our understanding of the computing process.

The digital technology evolution begins with the advent of the first digital programmed Unimate mechanism in 1954 (sold by General Motors in 1961). At first, robotic devices were intended for industrial production: the implementation of certain manipulations, navigation, moving heavy objects, etc. (McIlvaine Parsons \& Kearsley, 1982). Over time, the interest in digital development has increased the interest from the side of mass culture.

Robotics and artificial intelligence (AI) research made consistent attempts to create robots similar to humans, but these efforts had been limited mainly to mimicking human behavior, emotions, and physicality. The authors consider the experience of European and Eastern theater institutions in the use of robotic mechanisms as performing actors. Japanese researcher Sone, using the example of the comedy performance Bacarobo (directors Meiwa Denki and Yoshimoto Creative Agency (a part of Yoshimoto Kogyo), 2007-2008, Tokyo, Japan) explores the functional component of robotic mechanisms in the process of organizing a theatrical spectacle (2012). Kreutzer and Sirrenberg (2020), Knight (2011), and Myounghoon (2017) analyze the possibility of using robots in popular culture. At the same time, these issues are mainly debatable in nature. The studies of Paré (2015), Nishiguchi et al. (2017) indicate that robotic mechanisms on the stage can only perform programmed manipulations, while scientists Colton and Wiggins (2012), Bruce et al. (2000) insist on the autonomy and the possibility of android "improvisation" in stage space.

At the same time, studies of the similarity between robotic and human activities and the person (viewer's emotional response to such objects appear in the scientific literature). According to concept uncanny valley by Japanese robotics scientist and engineer Mori (2012), robots are attractive until the moment they are not able to completely imitate a person. This concept was also explored in the recently published papers by Burleigh et al. (2013), MacDorman and Chattopadhyay (2016, 2017), and Tinwell et al. (2011). MacDorman and Ishiguro (2006, p. 302) point out that "the valley means the level of kinship of a human observer to a similar copy - a copy of a person". In the contest of the article under study, a copy of a person is a robot. Ferrari, Paladino, and Jetten found that the humanoid creation led to an increased sense of threat to human identity. The more a robot resembles a real person, the more it "challenges" (the author's expression) human identity (Ferrari et al., 2016). Kaplan stated that these new machines challenge human uniqueness (2004).

Analysis of the literature on the chosen topic indicates the presence of different opinions on the problem of introducing AI technology into the stage space, the principles of organizing robotic performances, the possibility of human-android interaction on the stage and the role of mechanized "actors" in the process of creating a theatrical show. These specific questions indicate an urgent need for a study on the features of the functioning of robotic 
actors in theatrical performance and the interaction of an android with a human partner and the audience.

Achieving this goal involves solving the following interrelated tasks:

- to formulate the main conceptual and categorical apparatus on this topic;

- to determine the functional component of the activities of mechanized organisms (robots) regarding their possible use in theatrical and entertainment events;

- to trace and summarize the experience of using AI in the organization of theatrical and entertainment events.

\section{Features of the robot functioning in the stage space}

With the robot appearance on the scene, its functional component has changed. From the category of a robotic assistant capable of performing heavy physical operations, the android goes into the category of a stage partner. Regarding this, the robot needs to learn how to interact and communicate with a human.

The first robots' performances were a gimmick for the audience, so they were often based on a fantastic plot. For example: the world's first robot theater was opened in 2010 at the Copernicus Science Center (CSC) in Warsaw, Poland with a fantastic performance called Mortal Engines (director Paweł Kolanowski, 1992) based on the story of the same title by a Polish science fiction writer Stanisław Lem (1992). The theater repertoire includes several performances with the participation of mechanized actors: among them The Secret of the Empty Wardrobe (director Kolanowski, 2010) or The Ghosts of the Fourth Dimension (director Kolanowski, 2010) based on Flatland: A Romance of Many Dimensions by Abbott (1992), Prince Ferrix and Princess Crystal (director Kolanowski, 2011) based on the story of Lem (1974). The surreal storyline of the play Prince Ferrix and Princess Crystal was written specifically for robot actors. The main character, the robot Ferrix, falls in love with the Crystal robot. However, the heroine dreams of falling in love with a human, and ignores all robots competing for her attention.

"The works play beautifully, although the whole technology of their actions is seen firsthand. You can hear how the motors work, but at the same time, thanks to the voices of the wonderful actors, life is in them. Robots express feelings with a play of light, gestures; their emotions are visible in large screens-eyes. Just like people's" -

said the director of the CSC Robert Firmhofer (Legierska, 2014). The first robots were programmed before the start of the theatrical performance and performed the necessary manipulations at certain points in the stage performance. In the twenty-minute performance $I$, Worker (director Hirata, 2008, Osaka, Japan) based on the play by Japanese playwright Hirata (see Poulton, 2019), two robots played, programmed to pronounce the text at specific moments, move around and perform certain manipulations in accordance with the performance storyline. However, this case showed that at that time the robot could not be compared to a human being in performing abilities. Indeed, acting involves the ability to convince the viewer of the veracity and realism of the created acting image. The mechanized organism could not fulfill this function, but it exerted an extraordinary entertainment and originality in delivering the play's informative material. 
In 2014, the British company Engineered Arts Limited, run by designer Will Jackson, created RoboThespian, the robot actor, which, unlike its predecessors, has become more realistic and human-like by several criteria. Firstly, thanks to the combination of sensors and animatronics, robot can scan the body language, age, emotional state of the people around it and react accordingly. Secondly, the liquid crystal eyes and LED lighting in the RoboThespian package provide a realistic and at the same time fantastic effect of the acting. Thirdly, the robot can read the text and add the appropriate facial expression, sing, dance, without experiencing a phase of fear. Fourthly, with the help of the motion capture program, the mechanism can imitate the other person's movements and make eye contact with a stage partner.

The RoboThespian appearance on the stage marked the beginning of a new stage in the development of robotic theater and provided new opportunities for the interaction of an actor and android on the stage.

Due to RoboThespian, it became possible to implement more complex artistic techniques and directing-scenario ideas. Android can perform complex tasks, mimic human behavior, and it increasingly attracts viewers' attention.

The RoboThespian use of robots on stage can be seen also in the play The Sinister Valley by Francesca Talenti (2013, Chapell Hill, North Carolina, United States). The fantastic story is based on the story of a person who agrees to transfer his personality to a robot in exchange for money. Therefore, the use of robots in a stage production is fully justified by the development of events in the play. The aspiring director himself admitted that working with the technology of AI was not easy due to the untimely reaction and unnatural actions of the robotic performer.

The Carnegie Mellon University (CMU) project (1979-2020) shows that robots can operate autonomously and sometimes unpredictably for a director's group and audience. The developers tried to create robots that could improvise, instead of carrying out predetermined manipulations. According to the researchers, the dramatic plot is developed not due to the actions of the characters, but rather due to the goals and objectives (Bruce et al., 2000). No matter how the actor acts, the main thing is the realization of the goal. Therefore, robotic actors were programmed only for the final result, which they had to implement, overcoming certain obstacles. However, these barriers were different each time, so the robot could not repeat its actions of each performance, which means it worked autonomously and mostly unpredictably for the audience.

\section{Robotic imitation of playing musical instruments}

Often in the stage space robotic equipment is organized in the form of a visual installation. Japanese designer Yuri Suzuki, known for his work Will.i.am (Pyramidi) (2014, London, United Kingdom (UK)), has developed three unique robotic instruments that reproduce the sounds of the piano, guitar and drum. Each piece of equipment is built according to the principle of its musical protagonist. The first contains keys that are programmed to be pressed at the right time. Thus, at the time of the presentation of a robotic game, a person does not interact with the equipment. The second one contains strings; the third one contains 
drums. The principle of operation of the other structures is the same. The ensemble of these instruments without human help creates an incredible play, which is accompanied by a video projection. Using the example of this installation, the author shows that this kind of action can be reproduced exclusively with the help of technology, without human interference.

In installations A3 K3 (2017, Linz, Austria) by Dragan Ilić (Kreutzer \& Sirrenberg, 2020), Paul by Patrick Tresset (2016, London, UK) and the Painting Fool by Colton (2006, London, UK) robots create paintings online (Colton \& Wiggins, 2012). Robot Paul can scan a human face and, like a skilled artist, draw it on canvas, but he cannot reflect a certain emotion on the face. On the other hand, another Living Fool robot can make a drawing, imitating a certain mood programmed previously by a human. Researchers have programmed about fifty artistic styles in the database of the invention and accordingly designated each of them with the keyword emotions.

Now with the help of robotic technology, which is often called AI, it is possible to create theatrical shows, performances, musical presentations. At the same time, robotic mechanisms can perform functions of a team member, a performer, and an actor. An example is the mechanical music group Compressorhead (created by Frank Barnes, Markus Kolb, Stock Plum, John Wright and Rob Wright, 2013, Berlin, Germany), which consists exclusively of robots. The project was developed with the participation of German engineers in 2013 (Figure 1). The rock group has only three works: the "drummer" robot, which has four mechanized arms and two mechanized legs, works on fourteen drums kit, the "guitarist" with two arms and seventy-eight fingers, and the robot "vocalist" who can change tones and registers of its voice.

The example of the play - of robotic performers shows that androids can not only imitate human activity, but also significantly technically prevail over human performance skills, having significantly more technical fingers, hands, strings, and so on. However, robots do not have the ability to independently create musical parts and improvise like real rock performers. They only reproduce sound data created in advance by man. Therefore, the androids'

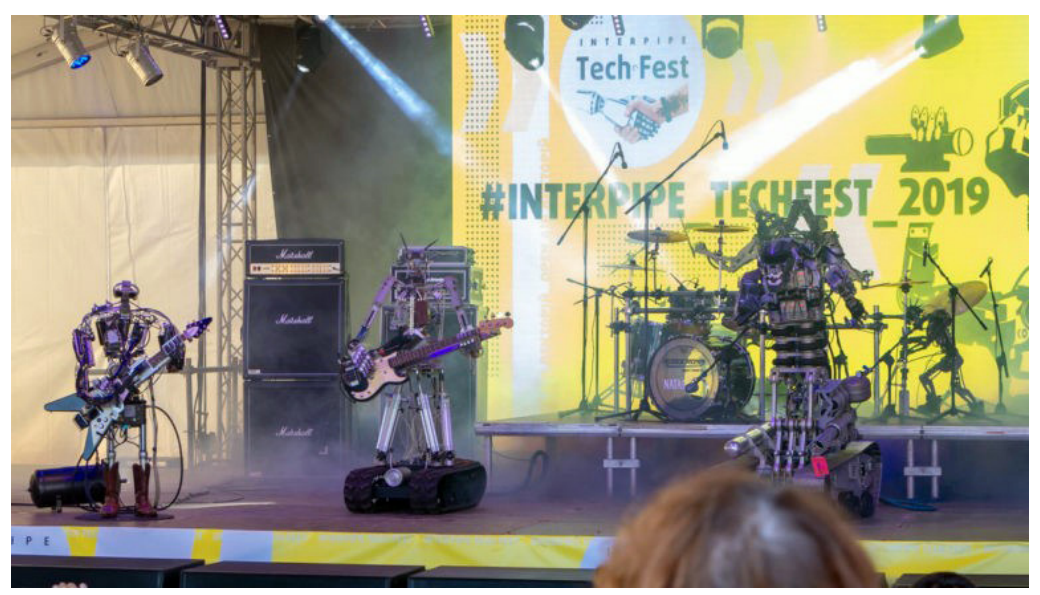

Figure 1. Mechanical music group Compressorhead (2013, Berlin, Germany) in Dnipro, Ukraine in 2020 (source: created by author) 
performance is of presentation nature. It is devoid of the properties of artistry, interactivity, and spontaneity. There is no creative improvisation process in this performance.

\section{Interaction features between a robot and a person in a stage space}

Research thought takes the next step, moving from analyzing specific features of the robots' performance in the stage space to clarify deeper, art aspects of the issues, including the analysis of the possible robotic organism communication and interaction with a real actor on stage.

At present, there have already been several projects in which the illusion of direct communication between the actor and the robot has been created. Japanese director Ishiguro in the experimental theater project Actors-Robots (2007) tried to show how people and mechanisms could reflect on socio-cultural problems in the future (Paré, 2015). It is curious that in this performance the robots had to "play" various roles (robot-"man", robot-"animal", etc.) and actively interact with partners and the environment (do a handshake, play football, return to the interlocutor, change locations) almost for the first time. Thus, in action, the illusion was created that robots can have partial free will and autonomy (improvisation). According to the developers Ogawa, Taura, and Ishiguro, the audience was more impressed by the performance of androids than the numbers of real actors (2012). We observe similar experimental attempts at interaction, communication, and even love twists and turns between the actor and the machine in the play Spillikin, A Love Story (director Natalie Gershtein, 2015) of the Pipeline Theater in Edinburgh, Scotland, UK.

Scientist Knight identified critical components for better programming of robots for theatrical stage: the implementation of appropriate gestures, expression of emotions, the metaphor of movement, communication with external emotions, social intelligence, feedback from the audience and machine humor. Robots should be able to perform movements and change their voice intonation to better convey the corresponding emotions (Knight, 2011).

The analysis of the specifics of the functioning of RoboThespian revealed that the operation of a modern robotic mechanism has several advantages over human performance.

Firstly, the functional component of the invention is more efficient and economical - it does not need breaks for sleep, food or rest.

Secondly, the results of this humanoid parameter analysis give reason to argue that with the 1.75 meters height, it is lighter $(33 \mathrm{~kg})$ than a human being and can be programmed in 30 different languages, which is extremely difficult for a human to do. It has an Atom $6 \mathrm{GHz}$ processor and a $32 \mathrm{~GB}$ solid-state drive, and a built-in $20 \mathrm{~W}$ sound amplifier allows you to hear your voice well without using sound amplifiers.

Thirdly, it turns out that the robot can carry out more than 30 variations of movement and emotions, sufficiently complements the text.

Fourthly, the robot can work in all weather conditions (even in the rain).

Fifthly, most importantly, the modern version of Engineered Arts Limited can keep eye contact with the viewer and interlocutor. Due to this function, it is endowed with the telepresence feature. 
Sixthly, the robotic mechanism is characterized by the ability to work autonomously. Autonomy is a characteristic that represents self-control and regulation of one's behavior in accordance with environmental conditions due to the ability to scan the space and faces around it, and an android can change its behavior depending on changes of the environmental conditions (Reeve, 2009). RoboThespian is an Internet-connected device that can be controlled via the online interface. The robot controller can be far away from the android. The robot can also independently interact with people, finding answers to web requests; can control the theater lighting and multi-channel sound.

Seventhly, a robot actor can look like any character or famous actor.

However, the cost of such experiments is extremely high. In my opinion, the reasons for the manifestation of the AI introduction in the theatre are rare. At present one robot costs more than eighty thousand dollars.

Recent experimental studies show that robots can instantly react to the actions of an actor on stage, interact with him and even choose the most correct movement option using algorithmic computation. As a result of 16 scenes and 40 demonstrations of movements, it was found that the performers and the audience perceived the robot as a stage partner (Rond et al., 2019).

\section{Role of a robot in creating a creative process}

Despite considerable interest and the first successful attempts at stage interaction between an actor and a robot, robotic performances have an ambiguous effect on the viewer. Many observers are suspicious of robots and do not identify the robot as an actor. This can be due to many factors. First, the unpredictability of the robot's actions makes the viewer feel stressed. According to the "threat perception" theory proposed by Kang (2001), robots put us in a state of cognitive dissonance, which entails a sense of fear of the unknown. In the scientific literature, there are more and more arguments about the technological singularity.

With technological progress, it is likely that robots will soon become as humanoid as possible. The audience will not be able to distinguish between a real actor and a robot. A similar situation is observed with the introduction of holographic projection into the stage space. But, if in the case of a projection, only the illusion of the presence of an object in space and interaction with a human being is created, then real communication with a robot is possible and even, as discussed above, improvisation (variability) of actions. The result is an uncanny valley effect discovered by Japanese robotics scientist and engineer Mori. The term "bukimi no tani genshō" was first translated as supernatural valley in the work Robots: Fact, Fiction, and Prediction by author Reichardt (1978). A theory is a person's response to the plausibility of an artificially created object as it approaches the real source. According to this concept, a robot or other object that looks or acts roughly like a human (but not exactly like a real one) causes dislike and disgust among human observers (Mori, 2012). The most humanoid robots suddenly turn out to be unpleasant for humans due to small inconsistencies in reality that cause feelings of discomfort and fear. Examples of the uncanny valley effect can be found in robotics, 3D modeling computer animation, and humanoid doll manufacturing. For clarity, Mori presented the following hypothetical graph. 
The graph shows that a gradual increase in attractiveness as the creature becomes more similar to a person is replaced by a sharp dip - the uncanny valley. Note that moving robots can generate stronger feelings of attraction and rejection.

One hypothesis is that an uncanny robot elicits an innate fear of death and culturally supported defenses for coping with death's inevitability (Burleigh et al., 2013).

There are several reasons for fear of mechanized organisms, as well as robotic technologies:

1. Since most androids are copies of real people, they cause fear in viewers to be replaceable in workplaces, in relationships and the like. Let us recall the historical facts that testify the people technophobia: the technical revolutions of the working population, the mass machines destruction;

2. In the functioning of a humanoid, a person can notice "defects" - differences in the behavior of a mechanized organism from human behavior, are perceived as a deviation and seem to indicate a disease of a mechanized organism (Rhodes \& Zebrowitz, 2002);

3. The humanoid mechanism is a soulless organism, it is not a creature. As in the case of the Golem in Judaism, whose lack of human empathy and spirit can lead to disaster, the android can pose a threat to society;

4. The idea of creating humanoids contradicts the position of existentialism - the philosophical trend of the 20th century, focuses its attention on the human existence uniqueness. The existence of artificial but humanoid beings is seen as a threat to the concept of human identity. These thoughts are outlined in the work Existential Psychotherapy by psychiatrist Yalom (1980). The author explains that people create psychological defenses to avoid existential anxiety associated with death. One of these defenses, the author points out, is the irrational belief that aging and death as central preconditions of life apply to everyone else except yourself (Yalom, 1980). The emergence of a humanoid disrupts human notions of "peculiarity" and existential defenses, causing existential anxiety;

5. A humanoid figure makes movements or other kinds of manipulations, imitating human behavior, as a result of which a feeling of psychological discomfort is created in the minds of observers, in psychology it is called cognitive dissonance.

The above proves that the humanoid, by introducing the observer into a cognitive dissonance state, generates in him a fear and hostility sense. The analysis results of the uncanny valley can become the basis for the creation of films or horror games, stage performances scenes, installations and the like (Tinwell, 2015).

This effect is present in the solo performance Uncanny Valley directed by Stefan Kaegi (2019, Munich, Germany) (Rimini Protokoll, 2020). According to a reviewer survey, the play caused a negative reaction from some viewers with its almost realistic but imperfect visual representation of human characters. The work appearance on the stage caused a lot of discussion among contemporary theater critics, but they all insisted on the Uncanny Valley effect presence. The director deliberately made the robots different on both sides: on the one hand, the organisms were as similar to a person as possible, on the other hand, the director left them without a human appearance, exposing wires and microcircuits. In this way, the author compared the reactions of viewers to humanoids and robots that do not resemble 
humans. Research has shown that humanoids are more attractive on stage because they remind us of real "live" actors. And mechanized organisms (non-humanoids) are not attractive to the stage.

Based on the research of the theory of Mori and his followers, we will formulate several criteria for the use of androids in the stage space:

1. The robot should not only imitate human actions on the stage, but also possess a unique distinctive feature for the interest and sympathy of the audience;

2. Androids, which are most similar to a human being, in the stage space can be used to create frightening performances;

3. Rapid attempts to humanize a robot can lead to irreversible consequences: full empathy and distrust of the viewer to the robotic actor. Therefore, it is important to give the movements of the humanoid unique features in order to expressively distinguish it from other performers in the stage space.

However, during a COVID-19 pandemic, interest in robotics is growing.

The COVID-19 pandemic has negatively affected the mental health of people around the world. According to one research "the pandemic has exacerbated work-related stress in many people, which has negatively impacted mental health" (Oracle, Workplace Intelligence 2020). Given the shortage of mental health professionals and social workers in many countries, some experts believe that the use of robots can help clinics diagnose diseases, giving psychiatrists and psychologists more time to consult other patients.

Let us recall the first computer program based on AI technology ELIZA (Sovhyra, 2020), which seemed to parody a dialogue with a psychotherapist, implementing the technique of active listening. Today, more and more often psychotherapy sessions are carried out with the help of robots. Research by the Australian Center for Robotic Vision (Australia) and Queensland University of Technology (Brisbane, Queensland, Australia) has shown that social robots have tremendous potential to help people cope with depression, drug and alcohol addiction and eating disorders (Ortenzi et al., 2019). According to researchers at the Technical University of Munich (Germany) robots could also pave the way for new treatments, the ability to target hard-to-reach populations and improve patient response (Haddadin, 2014). In the course of the work, the researchers studied the ethical consequences of communicating with a robot psychologist and found that robots manipulate people much more easily than other people. Thus, today we are witnessing a transformation of the form of human-robot relationship: from hostility, fear of technological determinism to the need for communication, trust and conditional "mutual understanding".

\section{Conclusions}

As a result of the study, the author revealed the partial independence of robots programmed in advance by a specialist. Robotic mechanism autonomy is possible, because the latest models of robots are able to scan the space, objects around it and to react at the right time according to a certain algorithm. 
These features bring the android closer to the real actor in the stage space. Improvisation as a characteristic feature of acting will be a manifestation of the autonomy of robot actions and "reactions". The robotic mechanism in the stage space is an experimental research platform. The projects are aimed at exploring the possibilities of robotic technology functioning in scenic spaces, the specifics of the interaction of humans and mechanized activities, and at the same time, the study of social futurology issues. The author revealed that the directors of England (UK), Scotland (UK), Poland, Japan and China actively use the AI technology in stage productions. Despite the high cost of technical experiments, androids on the stage have significant prospects for implementation. The robot can fully function as a unique technical invariant of a performer's actor in a stage production or art installation.

At the same time, the author insists on using a robotic mechanism as an original technological partner on the stage. The robot should not mimic human behavior on the stage as much as possible; these attempts can lead to irreversible consequences. Based on the theory of uncanny valley, the author suggests that humanoids, similar to humans, will evoke feelings of empathy, cognitive dissonance and fear in the audience. In contrast, androids that are not human-like, technologically superior to them (they have more arms, fingers, legs, like Compressorhead robots) are attractive to audiences.

As a result of studying the functional activity of robotic mechanisms in theatrical performances, the author was able to identify two ways of using robots in the stage space.

The first approach is the use of androids programmed to precisely perform certain manipulations at the right time and in the right place according to the plot of the play. The main task for the robot is to perform human-like movements of the robot. Director Hirata programs and tunes robots (robovie) in detail before the start of theatrical performance, so that movements resemble human gestures and are as natural as possible.

The second principle involves the use of robots, which may have variability in certain tasks with the definition of the main goal. The CMU project shows that robots can work autonomously and even unpredictably for the viewer. The robot is able to independently choose one of several options for solving the proposed problem using algorithmic analysis. Robot actors are programmed only for the end result, which they must realize, overcoming certain obstacles. In this way, neither the viewer nor the partner can predict the next action of the android. This means that the robot on stage can operate autonomously and is largely unpredictable for the audience. In this case, an illusion of improvisation inherent in the artistic process appears in the robot's behavior.

Opposite concepts of human liking/dislike for humanoids are the result of the formation of philosophical trends of technophobia and technophilia. They have existed and developed over the years, having indisputable facts of the negative impact of robotic technology on the human condition (the uncanny valley effect) and, on the contrary, being used as a treatment for psychological diseases using active listening, introduction to theatrical performances, and so on.

\section{Acknowledgements}

I would like to express my gratitude to the scientific consultant, Honorary Academician of the National Academy of Arts of Ukraine, Doctor of Philosophy Serhyi Bezklubenko. 


\section{References}

Abbott, A. E. (1992). Flatland: A romance of many dimensions. In Ph. Smith (Ed.), Dover thrift editions. S. Appelbaum (General Ed.). Dover Publications, Inc.

Bruce, A., Knight, J., Listopad, S., Magerko, B., \& Nourbakhsh, I. R. (2000, 24-28 April). Robot improv: using drama to create believable agents. In Proceedings 2000 ICRA, Millennium Conference. International Conference on Robotics and Automation. Symposia Proceedings (Cat. No.00CH37065) (pp. 4002-4008). San Francisco, California, United States. Institute of Electrical and Electronics Engineers.

Burleigh, T. J., Schoenherr, J. R., \& Lacroix, G. L. (2013). Does the uncanny valley exist? An empirical test of the relationship between eeriness and the human likeness of digitally created faces. Computers in Human Behavior, 29(3), 759-771. https://doi.org/10.1016/j.chb.2012.11.021

Colton, S., \& Wiggins, G. A. (2012, 27-31 August). Computational creativity: the final frontier? In L. De Raedt, Ch. Bessiere, D. Dubois, P. Doherty, \& P. Frasconi (Eds.), ECAI'12: Proceedings of the 20th European Conference on Artificial Intelligence (pp. 21-26). Montpellier, France. IOS Press.

Ferrari, F., Paladino, M. P., \& Jetten, J. (2016). Blurring human-machine distinctions: anthropomorphic appearance in social robots as a threat to human distinctiveness. International Journal of Social Robotics, 8, 287-302. https://doi.org/10.1007/s12369-016-0338-y

Fishwick, P. A. (Ed.). (2006). Aesthetic computing. Massachusetts Institute of Technology. https://doi.org/10.7551/mitpress/1135.001.0001

Haddadin, S. (2014). Springer tracts in advanced robotics ' 90 . Towards safe robots: approaching Asimov's 1st Law. Springer-Verlag. https://doi.org/10.1007/978-3-642-40308-8

Kang, M. (2001). The use of dreaming for the study of history. Rethinking History, 5(2), 275-283. https://doi.org/10.1080/13642520110052657

Kaplan, F. (2004). Who is afraid of the humanoid? Investigating cultural differences in the acceptance of robots. International Journal of Humanoid Robotics, 1(3), 465-480. https://doi.org/10.1142/S0219843604000289

Knight, H. (2011, November). Eight lessons learned about non-verbal interactions through robot theater. In B. Mutlu, Ch. Bartneck, J. Ham, V. Evers, \& T. Kanda (Eds.), Lecture Notes in Computer Science: Vol. 7072. Proceedings of Social Robotics: Third International Conference, ICSR (pp. 42-51). Amsterdam, The Netherlands. Springer-Verlag. https://doi.org/10.1007/978-3-642-25504-5_5

Kreutzer, R. T., \& Sirrenberg, M. (2020). Management for professionals. Understanding Artificial Intelligence: fundamentals, use cases and methods for a corporate AI journey. Springer Nature Switzerland AG. https://doi.org/10.1007/978-3-030-25271-7

Legierska, A. (2014). The rise of robotic theatre. In Culture.pl. https://culture.pl/en/article/the-rise-ofrobotic-theatre

Lem, S. (1992). Mortal engines. Harcourt Brace Jovanovich, Inc.

Lem, S. (1974). Prince Ferrix and the Princess Crystal. In The Cyberiad (pp. 283-295). Book/Harcourt, Inc.

MacDorman, K. F., \& Chattopadhyay, D. (2017). Categorization-based stranger avoidance does not explain the uncanny valley effect. Cognition, 161, 132-135.

https://doi.org/10.1016/j.cognition.2017.01.009

MacDorman, K. F., \& Chattopadhyay, D. (2016). Reducing consistency in human realism increases the uncanny valley effect; increasing category uncertainty does not. Cognition, 146, 190-205. https://doi.org/10.1016/j.cognition.2015.09.019

MacDorman, K. F., \& Ishiguro, H. (2006). The uncanny advantage of using androids in cognitive and social science research. Interaction Studies, 7(3), 297-337. https://doi.org/10.1075/is.7.3.03mac 
McIlvaine Parsons, H., \& Kearsley, G. P. (1982). Robotics and human factors: current status and future prospects. Human Factors: The Journal of the Human Factors and Ergonomics Society, 24(5), 535-552. https://doi.org/10.1177/001872088202400504

Mori, M. (2012). The uncanny valley. In Institute of Electrical and Electronics Engineers Robotics and Automation Magazine, 19(2), 98-100. https://doi.org/10.1109/MRA.2012.2192811

Myounghoon, J. (2017). Robotic arts: current practices, potentials, and implications. Multimodal Technologies and Interact, 1(5). https://doi.org/10.3390/mti1020005

Nishiguchi, Sh., Ogawa, K., Yoshikawa, Y., Chikaraishi, T., Hirata, O., \& Ishiguro, H. (2017). Theatrical approach: designing human-like behaviour in humanoid robots. Robotics and Autonomous Systems, 89, 158-166. https://doi.org/10.1016/j.robot.2016.11.017

Ogawa, K., Taura, K., \& Ishiguro, H. (2012, 9-13 September). Possibilities of androids as poetry-reciting agent. In Proceedings of 2012 Institute of Electrical and Electronics Engineers RO-MAN: The 21st Institute of Electrical and Electronics Engineers International Symposium on Robot and Human Interactive Communication (pp. 565-570). Paris, France. Institute of Electrical and Electronics Engineers. https://doi.org/10.1109/ROMAN.2012.6343811

Oracle; Workplace Intelligence. (2020). As uncertainty remains, anxiety and stress reach a tipping point at work: artificial intelligence fills the gaps in workplace mental health support. Al@Work Study 2020. https://www.oracle.com/a/ocom/docs/oracle-hcm-ai-at-work.pdf

Ortenzi, V., Controzzi, M., Cini, F., Leitner, J., Bianchi, M., Roa, M. A., \& Corke, P. (2019). Robotic manipulation and the role of the task in the metric of success. Nature Machine Intelligence, 1, 340-346. https://doi.org/10.1038/s42256-019-0078-4

Paré, Z. (2015). Robot actors: theatre for robot engineering. In Ehwa Institute for Humanities Science and LABEX Arts-H2H Laboratory (Eds.), Theatres du Posthumain (pp. 143-162). Arcarnet.

Poulton, M. C. (Ed.). (2019). Oriza Hirata: Citizens of Tokyo: Six Plays. Seagull Books.

Reeve, J. (2009). Understanding motivation and emotion. John Wiley \& Sons, Inc.

Reichardt, J. (1978). Robots: fact, fiction, and prediction. Penguin Books.

Rhodes, G., \& Zebrowitz, L. A. (Eds.). (2002). Facial attractiveness: evolutionary, cognitive, and social perspectives. In G. Rhodes (Series Ed.), Advances in visual cognition. Ablex.

Rimini Protokoll. (2020). Uncanny valley by Stefan Kaegi. https://www.rimini-protokoll.de/website/en/ project/unheimliches-tal-uncanny-valley

Rond, J., Sanchez, A., Berger, J., \& Knight, H. (2019, 14-18 October). Improv with robots: creativity, inspiration, co-performance. In Proceedings of 2019 28th Institute of Electrical and Electronics Engineers International Conference on Robot and Human Interactive Communication (RO-MAN). New Delhi, India. https://ieeexplore.ieee.org/stamp/stamp.jsp?arnumber=8956410\&casa_token=_NL0fNWHD X0AAAAA:Rk34broP7pPHH7IdD2CGDtl-22XPNIe10yzy2KK0tPMKoKmjEUK47W9CxuqC4IFTza5HJ9Tze_15

Sone, Y. (2012). Double acts: human-robot performance in Japan's Bacarobo Theatre. In G. Arrighi \& V. Emeljanow (Eds.), A world of popular entertainments: an edited volume of critical essays. Cambridge Scholars.

Sovhyra, T. (2020). AI technology and biological research use in cultural practices. International Journal of Recent Technology and Engineering, 9(4), 126-130. https://doi.org/10.35940/ijrte.D4880.119420

Tinwell, A. (2015). The uncanny valley in games and animation. A K Peters/CRC Press. https://doi.org/10.1201/b17830

Tinwell, A., Grimshaw, M., \& Williams, A. (2011). The uncanny wall. International Journal of Arts and Technology, 4(3), 326-341. https://doi.org/10.1504/IJART.2011.041485

Yalom, I. D. (1980). Existential psychotherapy. Basic Books. 